\title{
Correction procedures in extinction of matching behavior
}

\author{
GARY L. HOLT \\ Eastern Ilinois University, Charleston, Illinois 61920
}

\begin{abstract}
Three experiments using two pigeons were designed to assess correction procedure functions in acquisition and extinction of matching behavior. Experiment $\mathrm{I}-\mathrm{The}$ removal of correction procedures from blue and amber center key conditions during operation extinction produced sustained chance matching. Red center key conditions, not on operational extinction, remained at high baseline values. Experiment II-The occurrence of correction procedures for blue and amber center key conditions during operational extinction resulted in only temporary decrements in matching accuracy for blue and amber conditions. The removal of incorrect red center key correction responses slightly increased between-session variability in accuracy on red center key conditions. Experiment III-the percent correct responses to red center key conditions became more variable between sessions under baseline and extinction sessions following removal of correction procedures from all conditions. Blue and amber center key accuracy decreased, and maintained near chance performance.
\end{abstract}

Classical extinction (withdrawal of reinforcement) of a complex operant involving conditional discriminations (e.g., matching to sample) has been investigated by many researchers (Cohen, 1969; Cumming, Berryman, \& Cohen, 1967; Cumming, Berryman, \& Nevin, 1965; Jenkins, 1961). In these experiments operational extinction consisted of reinforcement withdrawal as a consequence for correct center side key matching responses on all stimulus conditions within the stimulus condition sequence. The operational extinction procedure, in these experiments, resulted in a decrease in the rate of matching until total extinction of all matching responses occurred within one or two sessions. Although rate decreased, matching accuracy did not. The question still remains open as to whether rate only and not accuracy of matching results from reinforcement withdrawal. The procedure in a later experiment was designed to decrease matching accuracy for those stimuli under operational extinction while maintaining high center side key response rates in the matching task (Holt \& Shafer, 1971). Correct center side key matching responses to stimuli not under operational extinction were maintained with high matching accuracy and rate. In this experiment responses to stimuli under operational extinction decreased in accuracy for the first few sessions, but subsequently increased to slightly below baseline acquisition performance thereafter. The increase in accuracy under operational extinction may have been due to a correction procedure following incorrect matches regardless of center key due. The operations used in the following three experiments were designed to assess the function of correction procedures in acquisition and extinction of matching behavior and to reaffirm that extinction of matching behavior can be reliably repeated.

\section{METHOD}

\section{Subjects}

Two White King pigeons approximately 3 years old were maintained within $15 \mathrm{~g}$ of $80 \%$ free-feeding weights on a grain mixture consisting of $62 \%$ cracked corn, $19 \%$ wheat, $18 \%$ milo, and $1 \%$ oats.

\begin{abstract}
Apparatus
A BRS-Foringer pigeon test chamber contained three plastic translucent pecking keys transilluminated by three IEE light projecting display cells (Model 1346). A filtered air intake and exhaust fan served as masking noise. All aspects of the experimental procedure were automatically controlled by relay circuitry.
\end{abstract}

\section{Procedure}

Preliminary training. Following magazine training the response requirement was increased to fixed ration five (FR5) on the center key, which was alternately illuminated with three hues (amber, red, and green). Responses to the illuminated key were reinforced with a $3-\mathrm{sec}$ grain magazine presentation. After the pecking response was well established on the center key, pecks to any illuminated, randomly presented, comparison key were reinforced.

Preliminary training was terminated when subjects pecked either illuminated side key with short latency, regardless of hue. On the following day, the simultaneous matching procedure was employed. The simultaneous matching procedure involved a 25-sec intertrial interval (ITI) after correct responses and a 25 -sec ITI and stimulus condition representation after incorrect responses.

\section{EXPERIMENT I: RED CORRECTION ONLY}

\section{Procedure}

During acquisition a correct response was followed by reinforcement then an ITI, while an incorrect response was followed by an ITI, then representation of the same condition. Acquisition was terminated after four consecutive sessions of approximately $85 \%$ matching had been established on most stimuli within the sequence. Following stable responding, operational extinction was begun. 


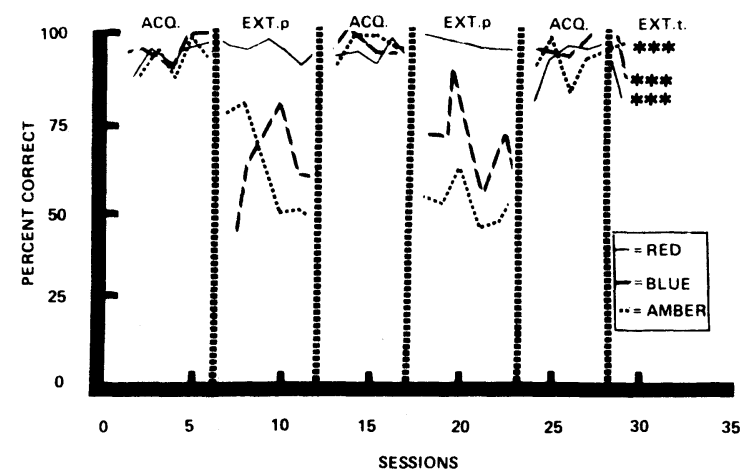

Figure 1. Percent correct matching red (solid line), blue (dashed line), and amber (dotted line) as a function of total acquisition and repeated acquisition (Acq.) and partial extinction (Ext.p.) treatments for red correction only. X's show incompleted sessions.

Operational extinction consisted of withdrawal of reinforcement as a consequence of correct matching responses to eight stimulus conditions (Conditions 4, 5, 6, 9-amber and 2, 7, 8,11 -blue), of the 12-condition sequence. The remaining 4 stimuli (Conditions 1, 3, 10,12-red) remained on an acquisition procedure while blue and amber were programmed for operational extinction. ${ }^{1}$ In addition, blue and amber conditions were programmed with no correction procedure following incorrect matching responses. After incorrect amber or blue responses, an ITI was followed by presentation of the next condition in the 12-condition sequence. The consequence of an incorrect red response was representation of that condition until a correct response was made. Six extinction sessions were run for each extinction treatment. A condition of total reinforcement withdrawal for correct responses to all stimuli was placed at the termination of each experiment. This total operational extinction procedure was continued until the subjects failed to respond for four hours or after four complete consecutive sessions. The procedure of acquisition extinction and reacquisition were repeated twice.

\section{RESULTS FOR EXPERIMENT I}

Since results of both Subjects 1 and 2 are similar in the following three experiments, only results of Subject 1 will be shown. Figure 1, Subject 1 shows percent correct matching on blue, and amber center key stimuli as a function of repeated baseline acquisition and extinction procedures and continuous acquisition procedures for red center key stimuli. Baseline, 85\%-100\% correct matching with all stimuli, regained reach within one or two sessions, and was maintained with low variability until the total extinction or probe operation was introduced at the end of the experiment.

Figure 1 shows functional differences between matching responses on both partial and total extinction operations. Percent correct matching on blue and amber decreased almost uniformly to chance levels, at approximately $50 \%$, under both partial extinction treatments. This decrement appears stable until the first session of each reacquisition operation. Percent of correct matching remained within baseline levels for two sessions of the total extinction operation. The last three

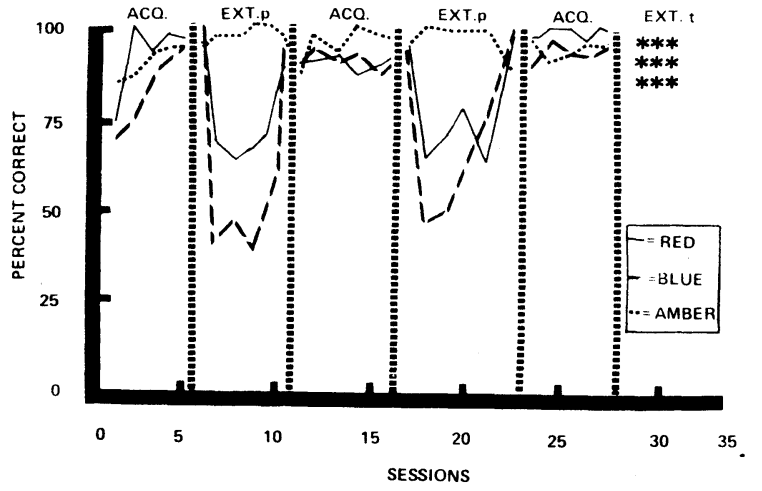

Figure 2. Percent correct matching red (solid line), blue (dashed line), and amber (dotted line) as a function of total acquisition and repeated acquisition (Acq.) and partial extinction (Ext.p.) treatments for blue and amber corrections only. X's show incompleted sessions.

sessions under total extinction went incomplete until the next reacquisition session. Correct matching responses to the four red center key stimuli maintained above $90 \%$ correct matching under all conditions. However, percent correct matching on blue and amber under the extinction treatment shows differences. In both extinction operations, matching responses on blue center key stimuli maintained higher mean percent correct matching performance, $63 \%$ and $76 \%$, than on amber center key stimuli, $66 \%$ and $55 \%$.

\section{EXPERIMENT II: BLUE AND AMBER CORRECTION ONLY}

In Experiment I a correction procedure, used with red stimuli and not used with blue and amber, produced no decrease in matching accuracy with red but produced a sustained decrease in matching accuracy with blue and amber conditions. It was also of interest to determine: (a) whether a correction procedure used only with amber and blue stimuli, and not with red stimuli, would produce lower than baseline acquisition percent matching responses with red stimuli conditions; and (b) whether blue and amber conditions with correction procedures would result in sustained decrements in matching accuracy under extinction operations.

\section{Procedure}

The procedure for the baseline acquisition was the same in this experiment as it was for Experiment I. The extinction procedure differed from Experiment I only in the following way: a correction procedure was used only for incorrect blue and amber responses, and not for incorrect red responses.

\section{RESULTS FOR EXPERIMENT II}

Figure 2, Subject 1, shows little difference in percent correct matching on blue and amber center key conditions as a function of repeated acquisition and extinction operations. Percent correct matching on red center key stimulus conditions show high perfrormance 
even though no correction procedure was programmed as a consequence of incorrect responses. Figure 2 shows high baseline percent correct matching on both amber and blue center key stimuli conditions for acquisition sessions. Response accuracy on blue and amber center key conditions decreased to or below chance levels under the extinction treatments during the first few extinction sessions. However, with continued sessions, matching accuracy returned to $90 \%$ matching for both blue and amber center key conditions.

\section{EXPERIMENT III: NO CORRECTION PROCEDURE}

Experiments I and II used a correction procedure during acquisition sessions. The extinction procedure of Experiments I and II involved a correction procedure programmed after only red conditions (Experiment I); and only blue and amber conditions (Experiment II). The purpose of the third experiment was to determine how the percent of correct matching responses would be affected by eliminating a correction procedure not only from the conditions under extinction but also under acquisition.

\section{Procedure}

The procedure used in this experiment was the same as was used for Experiments I and II except that no correction procedure was programmed for acquisition and extinction for red, blue and amber center key conditions.

\section{RESULTS FOR EXPERIMEN่T III}

Figure 3, Subject 1 shows percent correct matching on blue and amber center key conditions as a function of repeated acquisition and extinction operations, and percent correct matching on red conditions as a function of continuous acquisition. Responses to red stimulus condition treatments show more variability with lower percent matching under the acquisition sessions in Experiment III than in I and II. This effect was produced by the removal of all correction operations as a consequence of incorrect responses.

Figure 3 shows sharp decreases to chance level on amber stimulus conditions but decreases to only $70 \%$ correct for blue stimulus conditions under operational extinction. The performance under blue and amber stimuli in this experiment appears very similar to performance for the same stimuli in Experiment I.

\section{SUMMARY AND DISCUSSION}

It was found from a prior experiment (Holt \& Shafer, 1971) that operational extinction of 8 of the stimulus conditions (blue and amber) while maintenance of the remaining 4 stimuli conditions (red) on acquisition, resulted in functional extinction of percent correct matching on blue and amber conditions and baseline performance on the red center key stimulus conditions. However, the extinction operation involved a correction procedure contingent upon all incorrect responses. The consequence of the correction procedure resulted in an increase

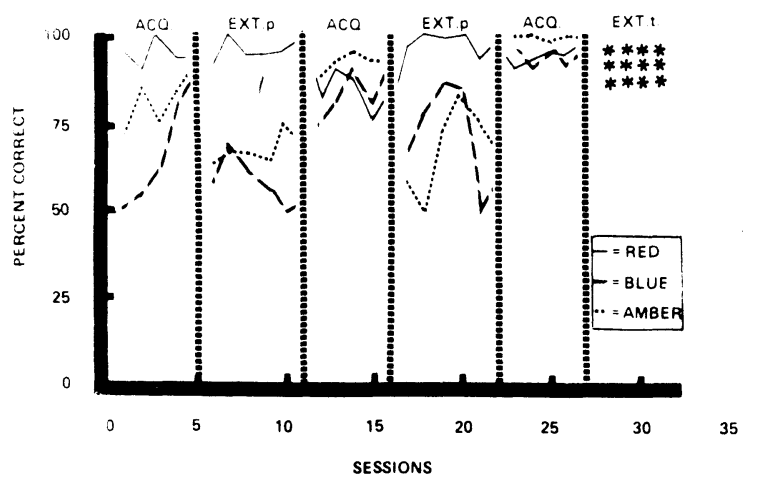

Figure 3. Percent correct matching red (solid line), blue (dashed line), and amber (dotted line) as a function of total acquisition and repeated acquisition (acq.) and partial extinction (Ext.p.) treatments with no correction procedure. X's show incompleted sessions.

in matching accuracy following an initial decrease to chance levels. The elimination of the correction procedure for incorrect responses to blue and amber center key stimuli, programmed in Experiment I above, produced sustained decrements in matching during the extinction operations.

It was not determinable from Experiment I whether the withdrawal of a correction procedure with its consequent increase in density of reinforcement was responsible for the sustained decrease in matching accuracy. Since a $25-\mathrm{sec}$ ITI was scheduled after each incorrect response in the correction procedure, each incorrect response consequently decreased the density of reinforcement. Since decreased reinforcement densities have been found to increase matching accuracy (Holt \& Shafer, 1973), it is possible that repeated ITI's following incorrect matchies may have produced the increase at the end of the extinction sessions. To determine what part of the extinction operation of Experiment I produced sustained decrements, additional extinction operations with and without an ITI must be compared.

The purpose of the second experiment was to determine the effect of: (a) no correction procedure after red center key stimulus conditions, and (b) a correction procedure for blue and amber conditions for extinction operations. The anticipated decrements in percent of correct matching of red stimuli under operational extinction did not occur. The maintenance of baseline performance of red stimuli may be due to the already high matching performance before initiation of the no correction condition during extinction. With such high preextinction matching, there is little opportunity for the no correction procedure to affect performance. Performance might decrease, however if extended sessions under the no correction procedure were initiated. The increase on blue and amber conditions, from chance matching to $90 \%$ accuracy under correction procedures, and operational extinction may be attributed to the issue of reinforcement density described above. Correction procedures appear to decrease reinforcement density thereby tending to increase matching accuracy.

For the third experiment, the effect of removal of a correction procedure for responses to all center key conditions as a consequence of incorrect responses was to produce more variability in both baseline acquisition treatments for red center key conditions than in Experiments I and II. The increased variability and decreased matching accuracy under red center key acquisition treatments and decreased variability and increased accuracy on blue and amber extinction treatments may suggest more support for a discussion of reinforcement density and reinforcement opportunity. The difference in accuracy between different session of the same red center key condition 
treatment may simply be explained as follows: withdrawal of a correction procedure for errors on all center key conditions increases reinforcement density or the opportunity to respond to and be reinforced for more correct matching-to-sample responses per unit of time. As has been stated elsewhere (Holt \& Shafer, 1973), this increase in reinforcement density effects on red center key matching was decreased under extinction operations due to the decrease in reinforcement opportunity per session. In order to maximize reinforcement opportunity per session, fewer errors may be made on red center key conditions when blue and amber conditions are under operational extinction. The results in Experiment III represents one of the only demonstrations of behavioral contrast in the accuracy of performance in a complex discrimination. Behavioral contrast occurred under conditions when reinforcement was no longer available for pecks to blue or amber and accuracy to red increased then decreased again when reinforcement was reintroduced for blue and amber responses. Further demonstration of this behavior is now being replicated.

In summary the results of the three experiments suggest: (1) that both rate and accuracy of matching behavior may be extinguished by different reinforcement withdrawal procedures; (2) that elimination of a correction procedure is sufficient to produce sustained decrements in matching accuracy under operational extinction procedures; (3) that the extinction of matching responses may be affected by reinforcement density of reinforcement opportunity; and (4) that the elimination of a correction procedure in acquisition and extinction operations results in increased variability and decreased accuracy.

\section{REFERENCES}

Cohen, L. R. Generalization during acquisition, extinction, and transfer of matching with an adjustable comparison. Journal of the Experimental Analysis of Behavior, 1969, 12, 463-474.

Cumming, W. W., Berryman, R., and Nevin, J. A. Search for an effect of satiation on delayed matching-to-sample performance. Psychological Reports, 1965, 16, 645-652.

Cumming, W. W., Berryman, R., \& Cohen, L., \& Lanson, R. N. Some observations on extinction of a complex discriminated operant. Psychological Reports, 1967, 20, 1328-1330.

Holt, G. L., \&Shafer, J. Function of intertrial interval in matching-to-sample. Journal of the Experimental Analysis of Behavior, 1973, 19, 181-186.

Holt, G. L., \& Shafer, J. Acquisition and Extinction of a Conditional Discrimination. Paper presented at the meeting of the Eastern Psychological Association, New York City, March, 1971 .

Jenkins, H. M. The effects of discrimination training or extinction. Journal of Experimental Psychology, 1961, 61, 111-121.

\section{NOTE}

1. For the stimulus condition sequence refer to the Journal of the Experimental Analysis of Behavior, 19, P. 182, 1973.

(Received for publication November 4,1974 .) 